\title{
E-Commerce: vom wirtschaftlichen Trugbild zum Gesellschaftswunder
}

Benoît Vulliet

\section{OpenEdition}

\section{Journals}

Electronic version

URL: http://journals.openedition.org/sjep/526

DOI: $10.4000 /$ sjep.526

ISSN: 1663-9677

\section{Publisher}

Institut de hautes études internationales et du développement

\section{Printed version}

Date of publication: 1 novembre 2003

Number of pages: $41-44$

ISSN: $1660-5926$

\section{Electronic reference}

Benoît Vulliet, «E-Commerce: vom wirtschaftlichen Trugbild zum Gesellschaftswunder »,

Schweizerisches Jahrbuch für Entwicklungspolitik [Online], 22-2 | 2003, Online erschienen am: 10 Juni

2010, abgerufen am 08 September 2020. URL : http://journals.openedition.org/sjep/526 ; DOI :

https://doi.org/10.4000/sjep.526 


\title{
E-Commerce: vom wirtschaftlichen Trugbild zum Gesellschaftswunder
}

\author{
Benoît Vulliet*
}

\section{Elektronischer Handel}

In Ländern, in denen die Bevölkerungsmehrheit über einen InternetZugang verfügt, ist der Glaube weit verbreitet, der elektronische Handel (E-Commerce) sei die Patentlösung für sämtliche Probleme der rund fünf Millionen Mikrounternehmen und KMU. Als Konsument findet der Internet-Nutzer mittlerweile ein schier unendliches Online-Angebot an Gütern und Dienstleistungen, so dass ein Leben ohne Internet kaum mehr vorstellbar scheint. Für Unternehmer stellt der elektronische Handel zunehmend ein unverzichtbares Hilfsmittel dar, sei es, um Produkte anzubieten oder um Transaktionen abzuwickeln.

Tatsächlich aber verfügen nur gerade $8 \%$ der Weltbevölkerung über einen Internet-Zugang, und 50\% der Nutzer leben in den reichen Ländern' ${ }^{1}$. Der elektronische Handel trägt lediglich $6 \%$ zum Welthandel bei. Von diesem entfallen auf die Entwicklungsländer weniger als $5 \%$, welche sich wie folgt auf die einzelnen Ländergruppen verteilen: Asien und Pazifik 83,5\%, Transitionsländer $8,7 \%$, Lateinamerika $7,3 \%$ und Afrika - der arme Verwandte $-0,5 \%$ (dies entspricht rund 500 Millionen USD) ${ }^{2}$.

Trotz seiner ausgeprägten Vielfalt ${ }^{3}$ entfällt der Grossteil des elektronischen Handels - nämlich 95\% - auf Transaktionen zwischen Unternehmen (B2B), und nur gerade $5 \%$ auf den Direktverkauf von Gütern und Dienstleistungen an die Verbraucher $(\mathrm{B} 2 \mathrm{C})^{4} . \quad \mathrm{Zu}$ den erfolgreichsten Akteuren im B2C-Geschäft zählen Online-Anbieter von Büchern, Videos und DVDs. In Entwicklungsländern ansässige Anbieter verkaufen vor allem Tourismusdienstleistungen oder handwerkliche Erzeugnisse. Da ihre Kundschaft mehrheitlich aus den Industrieländern stammt, betreiben sie auch ihre Server in der entwickelten Welt, um von den günstigen Rahmen-

* Lehrbeauftragter am Institut universitaire d'études du développement (iuéd), Schweiz.

1 Barayre-El Shami, Cécile, „Building Up New Business Models For Digital Inclusion“, ITU/IDSC Regional seminar on e-business for the Arab region, Kairo, Ägypten, 10.-12. Dezember 2002, $<$ www.ebi.org.eg/E>.

2 UNCTAD, E-commerce and Development Report 2002, S. 6-8. Gemäss diesem Bericht dürfte sich der Anteil des elektronischen Handels am Welthandel von 6\% im Jahr 2002 bis 2006 auf $18 \%$ erhöhen.

3 Von dieser Vielfalt zeugen zahlreiche Wortschöpfungen, wie zum Beispiel B2B (business to business, Transaktionen zwischen zwei Unternehmen, beispielsweise zwischen Herstellern und Zulieferern), B2C (business to consumer, Online-Verkauf von Gütern und Dienstleistungen direkt an die Endverbraucher), B2G (business to government, im Auftrag von Behörden tätige Unternehmen), C2C (consumer to consumer, Transaktionen zwischen Verbrauchern), usw.

$4 \quad$ UNCTAD, op. cit., S. 8. 
bedingungen wie Stabilität und Leistungsfähigkeit der Verbindungen sowie gesicherte Zahlungsdienstleistungen zu profitieren.

Der elektronische Handel beruht somit im Wesentlichen auf Handelsbeziehungen zwischen Unternehmen in den reichen Ländern, während er in anderen Regionen eine untergeordnete Rolle spielt. In den vergangenen Jahren hat sich jedoch eine neue Art von Unternehmen entwickelt, welche den Markt der Diaspora als bevorzugte Nische erschlossen haben: „B2D“ oder business to diaspora.

\section{Der Markt der Diaspora}

Der Markt der Diaspora wird von zwei Gruppen von Unternehmen bedient: Die eine Gruppe wird von Unternehmen gebildet, welche Güter und Dienstleistungen für in den Industrieländern lebende Auswanderer anbieten (business-to-diaspora consumer marketing $\left.{ }^{5}\right)$ : Reisen, Wohnungen, Geldüberweisungen, Nahrungsmittel, symbolische oder religiöse Gegenstände aus der Heimat. Zur zweiten Gruppe gehören Unternehmen, die in den Entwicklungsländern Produkte verkaufen und verteilen, welche von Auswanderern oder anderen mit dem Land verbundenen Personen für Familienangehörige und Freunde online eingekauft wurden. In diesem Fall sind an einer Transaktion drei Akteure beteiligt, was mit dem Begriff split-marketing ${ }^{6}$ umschrieben wird: Der Käufer im Industrieland, der Verkäufer im Entwicklungsland und der Empfänger im gleichen Ent- wicklungsland. Diese Unternehmen (d. h. die Verkäufer) werden zumeist von Personen geführt, die selbst eine gewisse Zeit zu Erwerbs- oder Ausbildungszwecken in einem industrialisierten Land gelebt haben und welche sich mit den erworbenen Fähigkeiten und Kenntnissen in ihrer Heimat eine Existenz gründen wollen. Namentlich Frauen nutzen die Gelegenheit, neben ihren Familienpflichten ein Unternehmen aufzubauen ${ }^{7}$.

Über ein peruanisches Unternehmen, welches Kuchen für festliche Anlässe verkauft, wurde Folgendes berichtet ${ }^{8}$ : „Selbst mit einem so einfachen Produkt wie selbstgebackenem Kuchen lässt sich ein Unternehmen im elektronischen Handel aufbauen, welches den gesamten inländischen Markt bedient. Einfache Hausfrauen lernen rasch, das Internet für die Entgegennahme von Bestellungen $\mathrm{zu}$ nutzen. Diese Frauen können neben der Kinderbetreuung das Internet nutzen und mit dem Backen Geld verdienen, ohne eine Arbeit ausser Haus suchen zu müssen. Mit dem Einzug von E-Mail und Computern verändert sich nicht nur die Welt, sondern auch das Gewerbe. Die Bevölkerung der Entwicklungsländer muss diese Gelegenheit ergreifen und ihre Phantasie und Kreativität nutzen, um Arbeitsplätze zu schaffen und Einkommen zu generieren. Dieses E-Commerce-Modell ist sehr einfach, lässt sich kostengünstig umsetzen und eignet sich auch für andere Länder und Produkte. Phantasie, Arbeitswille und Aufgeschlossenheit gegenüber der Technologie reichen aus, um Erfolg zu haben. ${ }^{69}$

5 Vgl. Economic Commission for Africa, The African Development Forum '99: Post ADF Summit. Electronic Commerce in Africa, 1999: <www.uneca.org/adf99/adf99ecommerce.htm>.

\section{Idem.}

UNCTAD, op. cit., S. 64.

<www.tortasperu.com.pe>. 
Aber ist die Umsetzung ebenso einfach wie das Prinzip? Sind diese Unternehmen wirtschaftlich überlebensfähig? Sehr oft waren sich diese Unternehmerinnen und Unternehmer nicht bewusst, welche Schwierigkeiten auf sie zukommen würden und welche Sachzwänge mit einer solchen Unternehmenstätigkeit verbunden sind.

\section{Schwierigkeiten und Lösungen}

Es handelt sich dabei um Sach zwänge, mit denen sich jedes Unternehmen im elektronischen Handel konfrontiert sieht, das Geschäftsbeziehungen in den Entwicklungsländern unterhält, nämlich :

- rechtliche und regulatorische Aspekte: Verschlüsselung/Entschlüsselung, digitale Signatur, Zertifizierungsorgane, Verbraucherschutz, lückenhafte Gerichtsbarkeit, geistige Eigentumsrechte, handelsbezogene Regeln und Verfahren.

$\checkmark$ finanzielle Aspekte: elektronischer Zahlungsverkehr, lokale Banken, welche zur Abwicklung der Transaktionen nicht bereit sind.

- technologische Aspekte: Telekommunikationsinfrastruktur (Netz und Bandbreite), Ausstattung der Nutzer (Haushalte und Unternehmen), hohe Verbindungskosten.

- logistische Aspekte: Zollabfertigung, Lagerung und Verteilung der Ware ${ }^{10}$.
Entscheidende Voraussetzungen für das split-marketing sind die Fähigkeiten, unterschiedlichste Produkte landesweit zu vertreiben und Zahlungen per Kreditkarte abzuwickeln, was die Wirtschaftlichkeit limitiert (zwischen 2000 und 30'000 USD pro Jahr) trotz der bedeutenden Summen, welche dank der Diaspora ins Land strömen (im Falle Äthiopiens beispielsweise 100 Mio. USD jährlich) ${ }^{11}$.

Zahlreiche Hürden und eine geringe Ertragskraft hindern diese Unternehmen nicht, sich weiterzuentwickeln und zu vermehren. Auf der Suche nach neuen Finanzierungsquellen wenden sich die Unternehmerinnen und Unternehmer an Ministerien, internationale und nationale Akteure der Entwicklungszusammenarbeit und Nichtregierungsorganisationen und verweisen auf den sozialen Nutzen ihrer Tätigkeit: Schaffung von Arbeitsplätzen, Ausbildung, Nutzung von Kompetenzen, Stärkung verwandtschaftlicher und zwischenmenschlicher Beziehungen. Die wirtschaftliche Stärke dieser Unternehmen beruht somit nicht in erster Linie auf den bedeutenden Finanzflüssen zwischen der Diaspora und der Heimat, sondern auf ihrer Fähigkeit, die soziale Dimension ihrer Tätigkeit auszuschöpfen.

Auch wenn der elektronische Handel angesichts des weltweiten Handelsvolumens insgesamt gesehen tatsächlich bedeutungslos ist, weist ein Unterneh-

9 „Ordinateurs et gâteaux donnent confiance et argent aux ménagères péruviennes“, in Forum du commerce international, La revue du Centre de commerce international (CCI), Nr. 3, 2000, S. 30. $<$ www.forumducommerce.org/news/fullstory.php/aid/169/>.

10 Vgl. Economic Commission for Africa, op. cit.

11 Barayre-El Shami, Cécile, „Information and communication technologies: Opportunities and challenges for SMEs in developing countries“, Latin American and Caribbean Symposium on Education, Science and Culture in the Information Society, Kuba, 18.-21. Februar 2002, 13 S.: $<$ http://espejos.unesco.org.uy/simplac2002/Ponencias/SIMPLAC/SL057.doc>. 
men, welches den Markt der Diaspora bedient, ein beträchtliches lokales Entwicklungspotenzial auf. Dieses Potenzial ist im Wesentlichen auf die Phantasie und den Erfindungsreichtum der Frauen und Männer zurückzuführen, die den Mut haben, ein Vor- haben mit signifikanter sozialer Wirkung zu realisieren. Offen ist nach wie vor die Frage, wie sich diese Wirkung manifestieren wird und welches ihre tatsächlichen Ursachen sind eine Frage, die von der Forschung beantwortet werden muss. 